\title{
Human papillomavirus in anal squamous cell carcinoma: an angel rather than a devil?
}

\author{
Paola Simona Ravenda ${ }^{1}$, Maria Giulia Zampino ${ }^{1}$, Nicola Fazio', Massimo Barberis ${ }^{2}$, Luca Bottiglieri ${ }^{2}$ and Susanna Chiocca ${ }^{3}$ \\ 'Unit of Gastrointestinal and Neuroendocrine Tumours, European Institute of Oncology, Via Ripamonti 435, 20141 Milan, Italy \\ ${ }^{2}$ Division of Histopathology and Molecular Diagnostics, European Institute of Oncology, Via Ripamonti 435, 20141 Milan, Italy \\ ${ }^{3}$ Department of Experimental Oncology, European Institute of Oncology, Via Ripamonti 435, 20141 Milan, Italy
}

Correspondence to: Paola Simona Ravenda. Email: paolasimona.ravenda@ieo.it

\begin{abstract}
Anal cancer is a rare disease with an increasing incidence worldwide but, unfortunately, even today the scientific community still has a limited knowledge and limited options of treatment.

More than $50 \%$ of patients with anal cancer presenting at diagnosis with locoregional disease have good chances of cure with chemoradiotherapy (CT-RT). However, once patients develop metastatic spread, the prognosis is very poor.

Human papillomavirus (HPV) is present in more than $80 \%$ of anal cancers and while multiple etiologic connections between HPV infection and anal cancer have already been well elucidated, its prognostic and/or predictive role is currently under investigation, especially among immunocompetent patients affected by this disease.

In a single-institutional set, we have retrospectively analysed clinical data of 50 consecutive cases homogeneously treated with CT-RT for stage I-III anal squamous cell carcinoma. We found that HPV-positive anal cancers had a statistically significant improved five-year disease-free survival (DFS) compared to HPV-negative group. These findings could be explained by an increased chemo/radiosensitivity of HPV-positive tumours. Further efforts should be directed towards a better understanding of HPV-related oncogenesis and towards designing novel tailored strategies for the management of this disease both in terms of prevention and treatment.
\end{abstract}

Keywords: anal squamous cell carcinoma, anal cancer, human papillomavirus, HPV-16

Published: 29/04/2015

Received: 04/11/2014

ecancer 2015, 9:529 DOI: 10.3332/ecancer.2015.529

Copyright: (c) the authors; licensee ecancermedicalscience. This is an Open Access article distributed under the terms of the Creative Commons Attribution License (http://creativecommons.org/licenses/by/3.0), which permits unrestricted use, distribution, and reproduction in any medium, provided the original work is properly cited. 


\section{Background and discussion}

Anal carcinoma $(A C)$ is a rare disease in the general population, being estimated at about 1.5 cases per 100,000 people per year worldwide, but with an increasing incidence particularly in women [1, 2].

Several biomolecular and epidemiologic studies have clearly elucidated a strong etiologic connection between AC and high-risk genotypes of HPV [3, 4], and indeed HPV infection is detected in between $86 \%$ and $97 \%$ of all AC cases [5].

Factors increasing the risk of HPV infection and/or modulating host response and the persistence of this infection appear to affect the epidemiology of this tumour. Anal intercourse and a high lifetime number of sexual partners increase the risk of persistent HPV infection in men and women, leading eventually to malignancy. Other important risk factors include human immunodeficiency virus (HIV), immunosuppression in transplant recipients, use of immnunosuppressants, a history of other HPV-related cancers, autoimmune disorders, social deprivation, and cigarette smoking [6].

Not surprisingly, AC is no longer a rare disease within specific epidemiologic categories, since among HIV seropositive men the incidence of $A C$ increases to 75-135 per 100,000 and is also higher among HIV seropositive women [6]. Therefore, in this regard, AC is one of the most representative examples of human cancers that emphasises how much the immune system and the process of tumourigenesis are inextricably linked.

On the other hand, few studies have so far evaluated the prognostic or predictive role of HPV in AC, especially among the general population and immunocompetent patients.

Based on these considerations we have retrospectively reviewed the data of a series of 50 consecutive and immunocompetent cases, treated in our institution between 2000 and 2012. These patients had AJCC (American Joint Committee on Cancer) stage I-III histologicallyproven anal squamous cell carcinoma, and had undergone standard treatment with concurrent chemo-radiotherapy (CT-RT). In all patients the presence of HPV infection was assessed by a polymerase chain reaction (PCR) analysis on the tumour biopsy provided at the time of diagnosis before treatment. Genomic DNA was extracted from three 10- $\mu$ m-thick paraffin sections using QIAamp DNA FFPE Tissue kit (Qiagen, Hiden, Germany), following the manufacturer's instructions, then 200 ng of DNA were amplified using L1 primers and GP5+/GP6+ primers. For all the PCR procedures, a positive and a negative control were provided. All the positive cases were confirmed in a second round of amplification. The PCR products were detected by electrophoresis with a $2 \%$ agarose gel and stained with ethidium bromide and in the presence of HPV infection, we proceeded to do the genotyping by testing the presence of high-risk HPV16, 18, 31, 33, and 45, using specific primers. Inclusion and exclusion criteria as well as the details on concurrent CT-RT are extensively reported elsewhere [7].

In our study we found that $84 \%$ of patients were HPV-positive and, among these HPV genotype 16 was detected in $90 \%$ of cases. HPV-positive patients had more advanced disease at diagnosis compared to HPV-negative patients in terms of nodal involvement affecting TNM clinical stage.

In particular, within HPV-positive group $2.4 \%$ of patients had stage I, $23.8 \%$ stage II, $45.2 \%$ stage IIIA, $28.6 \%$ stage IIIB, and within HPVnegative group $0 \%$ of patients had stage I, $62.5 \%$ stage II, $37.5 \%$ stage IIIA, $0 \%$ stage IIIB.

In this regard, in the statistical analysis, we decided to apply an adjustment for clinical stage in multivariate analysis since the HPV-positive patients presented a higher rate of nodal involvement [7].

After a median follow-up of four years, we found that five-year DFS in HPV-positive and HPV-negative patients was $92.5 \%$ and $50 \%$ respectively $(p<0.01$ ). Five-year overall survival (OS) in HPV-positive and HPV-negative patients was $93.3 \%$ and $66.7 \%$, respectively $(p=0.12)$. In conclusion, patients with HPV-positive AC had a strong statistically significant improved DFS compared to that in HPVnegative patients; the same trend was observed for OS, but statistical significance was not reached.

Despite its small sample size and retrospective nature, this study shows that HPV infection in AC is a positive prognostic factor for DFS. This could be explained by the better response of HPV-positive AC to CT-RT, as already shown in HPV-positive oropharyngeal squamous cell carcinoma [8, 9].

Certainly a greater patient sample size and longer follow-up might also show in the near future a significant difference in OS between HPV-positive and negative AC. 
From our perspective, the most intriguing and challenging question is whether HPV-positive AC and HPV-negative AC are two completely different malignancies sustained by different biomolecular features and genetic alterations. Further research should be directed towards investigating which specific pathways are selectively altered in anal cancers which are HPV-positive and those which are HPV-negative, by adopting a comparative approach. This should lead to a better understanding of HPV-related oncogenesis and to the design of novel and personalised tailored strategies for the management of this disease both in terms of prevention and treatment.

Preclinical models as faithful as possible to the true HPV-related tumour cell biology should be the right place from which experimental studies can embark upon their challenging road.

Currently the vast majority of modern studies, including analysis of E6 and E7 mRNA [10, 11], PIK3CA mutations [12, 13], and inhibition of aberrant cap-dependent protein translation $[14,15]$ are essentially applied to head and neck and cervical cancer models. Instead we believe that, because of shared underlying HPV biology, there is an opportunity for collaborative development of research strategies among investigators across the spectrum of HPV-related malignancy. Indeed in our centre we are building upon a new multidisciplinary approach in order to connect and guide scientific and clinical efforts within a common network for prevention and treatment of these diseases.

\section{Conclusion}

Despite its small sample size and retrospective nature, this study shows that HPV infection in anal cancer is a positive prognostic factor for disease-free survival. This could be explained by the better response of HPV-positive anal cancer to chemo-radiotherapy, as already shown in HPV-positive oropharyngeal squamous cell carcinoma.

From our perspective, the most intriguing and challenging question is whether HPV-positive anal cancer and HPV-negative anal cancer are two completely different malignancies sustained by different biomolecular features and genetic alterations. Further research should be directed towards investigating which specific pathways are selectively altered in anal cancers which are HPV-positive and those which are HPV-negative, by adopting a comparative approach. This should lead to a better understanding of HPV-related oncogenesis and to the design of novel and personalised tailored strategies for the management of this disease both in terms of prevention and treatment.

\section{Conflicts of interest}

The authors declare that they have no conflicts of interest.

\section{Acknowledgments}

The authors thank the patients as being their inspiration for this original article.

\section{References}

1. Johnson LG et al (2004) Anal cancer incidence and survival: the surveillance, epidemiology, and end results experience 1973-2000 Cancer 101(2) 281-8 DOI: 10.1002/cncr.20364 PMID: 15241824

2. Jemal A et al (2013) Annual report to the nation on the status of cancer, 1975-2009, featuring the burden and trends in human papillomavirus (HPV)-associated cancers and HPV vaccination coverage levels J Natl Cancer Inst 105(3) 175-201 DOI: 10.1093/ inci/djs491 PMID: 23297039 PMCID: 3565628

3. Frisch $\mathrm{M}$ et al (1997) Sexually transmitted infection as a cause of anal cancer N Engl J Med 337(19) 1350-8 DOI: 10.1056/ NEJM199711063371904 PMID: 9358129

4. Daling JR, Madeleine MM and Johnson LG (2004) Human papillomavirus, smoking, and sexual practices in the etiology of anal cancer Cancer 101(2) 270-80 DOI: 10.1002/cncr.20365 PMID: 15241823 
5. Human papillomavirus-associated cancers-United States 2004-2008 MMWR Morb Mortal Wkly Rep 2012 61(15) 258-61

6. Glynne-Jones R et al (2012) Anal cancer: ESMO-ESSO-ESTRO clinical practice guidelines for diagnosis, treatment and follow-up EJSO 40(10) 1165-76

7. Ravenda PS et al (2014) Prognostic value of human papillomavirus in anal squamous cell carcinoma Cancer Chemother Pharmacol 74(5) 1033-8 DOI: 10.1007/s00280-014-2582-x PMID: 25209946

8. Ang KK et al (2010) Human papillomavirus and survival of patients with oropharyngeal cancer N Engl J Med 363(1) 24-35 DOI: 10.1056/NEJMoa0912217 PMID: 20530316 PMCID: 2943767

9. Rischin D et al (2010) Prognostic significance of p16INK4A and human papillomavirus in patients with oropharyngeal cancer treated on TROG 02.02 phase III trial J Clin Oncol 28(27) 4142-8 DOI: 10.1200/JC0.2010.29.2904 PMID: 20697079 PMCID: 2953971

10. Rampias T et al (2009) E6 and E7 gene silencing and transformed phenotype of human papillomavirus 16-positive oropharyngeal cancer cells J Natl Cancer Inst 101(6) 412-23 DOI: 10.1093/jnci/djp017 PMID: 19276448

11. Sima N and Wang S et al (2007) Antisense targeting human papillomavirus type 16 E6 and E7 genes contributes to apoptosis and senescence in SiHa cervical carcinoma cells Gynecol Oncol 106(2) 299-304 DOI: 10.1016/j.ygyno.2007.04.039 PMID: $\underline{17586029}$

12. Nichols $A C$ et al (2013) High frequency of activating PIK3CA mutations in human papillomavirus-positive oropharyngeal cancer JAMA Otolaryngol Head Neck Surg 139(6) 617-22 DOI: 10.1001/jamaoto.2013.3210 PMID: 23787421

13. Lui VWY et al (2013) Frequent mutation of the PI3K pathway in head and neck cancer defines predictive biomarkers Cancer Discov 3(7) 761-9 DOI: 10.1158/2159-8290.CD-13-0103 PMID: 23619167 PMCID: $\underline{3710532}$

14. Spangle JM and Munger K (2010) The human papillomavirus type 16 E6 oncoprotein activates mTORC1 signaling and increases protein synthesis J Virol 84(18) 9398-407 DOI: 10.1128/JVI.00974-10 PMID: 20631133 PMCID: 2937655

15. Spangle JM, Ghosh-Choudhury N and Munger K (2012) Activation of cap-dependent translation by mucosal human papillomavirus E6 proteins in dependent on the integrity of the LXXLL binding motif $J$ Virol 86(14) 7466-72 DOI: 10.1128/JVI.00487-12 PMID: 22553330 PMCID: $\underline{3416298}$ 